(C) 2016, Elsevier. Licensed under the Creative Commons Attribution-NonCommercial-NoDerivatives 4.0 International http://creativecommons.org/licenses/by-nc-nd/4.0/

\title{
Developments in contact lens measurement: a comparative study of industry standard geometric inspection and optical coherence tomography
}

Coldrick, Benjamin J. ${ }^{1,2}$; Richards, Colin. ${ }^{2}$; Sugden, $\mathrm{K}^{3}$; Wolffsohn, JS. ${ }^{1}$;Drew, Thomas E. ${ }^{1}$

1. Aston University, Life and Health Sciences, Biomedical Engineering, Birmingham, United Kingdom

2. Optimec Limited, Malvern, United Kingdom

3. Aston University, Engineering and Applied Sciences, AIPT, Birmingham, United Kingdom

\section{Corresponding author:}

Benjamin Coldrick

b.coldrick1@aston.ac.uk

Present address:

Aston University, MB523 - Biomedical Engineering, Life \& Health Sciences, Aston Triangle, B4 7ET, United Kingdom

\section{Abstract:}

Purpose: The aim of this study was to compare a developmental Optical Coherence Tomography (OCT) based contact lens inspection instrument to a widely used geometric inspection instrument (Optimec JCF), to establish the capability of a market focused OCT system.

Methods: Measurements of 27 soft spherical contact lenses were made using the Optimec JCF and a new OCT based instrument, the Optimec is830. Twelve of the lenses analysed were specially commissioned from a traditional hydrogel (Contamac GM Advance 49\%) and 12 from a silicone hydrogel (Contamac Definitive 65), each set with a range of back optic zone radius (BOZR) and centre thickness (CT) values. Three commercial lenses were also measured; CooperVision MyDay (Stenfilcon A) in -10D, -3D and +6D powers. Two measurements of BOZR, CT and total diameter were made for each lens in temperature controlled saline on both instruments.

Results: The results showed that the is 830 and JCF measurements were comparable, but that the is 830 had a better repeatability coefficient for BOZR $(0.065 \mathrm{~mm}$ compared to $0.151 \mathrm{~mm})$ and CT $(0.008 \mathrm{~mm}$ compared to $0.027 \mathrm{~mm})$. Both instruments had similar results for total diameter (0.041 mm compared to $0.044 \mathrm{~mm}$ ).

Conclusions: The OCT based instrument assessed in this study is able to match and improve on the JCF instrument for the measurement of total diameter, back optic zone radius and centre thickness for soft contact lenses in temperature controlled saline. 


\section{Introduction}

With the increasing usage of contact lenses worldwide, the continual improvement of manufacturing methods and the increasing complexity of contact lens designs and materials, the inspection and quality control of contact lenses is paramount. Typically, inspection of contact lenses can be split into two broad areas: geometric inspection (e.g. curvature, centre thickness) and optical performance measurement (e.g. central and peripheral power). There are a wide range of methods available for geometric metrology, either included in the current ISO standards [1] (e.g. mechanical thickness gauges, optical projection techniques, ultrasound, v-gauges) or those more recently developed (e.g. low coherence interferometry [2], ptychography [3]). ISO standards are developed based on the available methods at the time of publication and as a result there are a number of potential shortcomings in the standard methods for contact lens inspection. Centre thickness measurement is conducted in air, resulting in a lack of appropriate temperature and hydration control, factors which can cause significant lens shape changes [4]; multiple instruments generally need to be utilised to obtain all the required measurements of a lens (e.g. measurement of sagittal depth [5], obtaining both centre thickness and curvature); there may be a large level of subjectivity in the measurements due to the usage of analogue measurement methods, and the time taken for measurement can be significant.

More importantly these methodologies only cover a small number of the wide range of clinically important parameters that are part of soft contact lens design and manufacture, with the standards principally covering centre thickness, posterior curvature (typically referred to base curvature or back optic zone radius, BOZR) and diameter. In particular, there is currently no standard for measuring key dimensional parameters at positions other than the centre of a lens, with emerging research showing the importance of measuring both sagittal depth[5] and thickness[6] at these positions, with peripheral shape becoming increasingly important for emerging lens designs such as those for myopia control[7]. As a result, there is an increasing need for instrumentation that can offer measurement of the key parameters across the entire lens, for all different lens designs and materials, with appropriate temperature control of the measurement medium.

One approach that may offer this functionality is optical coherence tomography (OCT), which has already been shown to be effective in measuring soft and rigid lenses [8-11]. To date, the application of OCT to contact lenses has utilised high-specification and therefore high-cost OCT systems. In addition these studies utilise methods that are impractical for usage in production, such as contrast enhancing agents in the measurement medium $[8,11]$ or complex lens positioning methods [11]. The purpose of the current study is to compare the measurement capability of an industry focused OCT contact lens instrument, with a standard geometric inspection instrument that is widely used in contact lens inspection to measure BOZR and Diameter, conforming to the current ISO standards.

\section{Methods}

The two instruments used in the study were the Optimec JCF and the Optimec is 830 development instrument (both manufactured by Optimec Limited, Malvern, UK). The JCF is a widely adopted projection based instrument; the is 830 is an OCT-based instrument that uses interferometry to produce an image of transparent or semi-transparent samples. Both instruments are described in more detail below. 


\section{Instrumentation - JCF}

The Optimec JCF is a projection based instrument incorporating two temperature controlled wet cells (in the current study controlled by an Optimec TC2Oi at $20^{\circ} \mathrm{C}$ to $\pm 0.5^{\circ} \mathrm{C}$ ). The first cell allows measurement of the lens diameter by placing a lens onto a graticule with the lens and graticule projected and magnified by $\times 17$ to allow measurement with a visual scale giving a resolution of $\pm 0.025 \mathrm{~mm}$ (Figure 1, B). The second cell uses a cylinder and probe to calculate the BOZR by utilising the sagittal height across a 10mm chord method (ISO 18369-3:2006 Section 4.1.4.2.2, [1]), with a resolution of $\pm 0.02 \mathrm{~mm}$. There is also a projected image (x17) of the lens (Figure 1, A) allowing the centre thickness to be estimated using a scale on the screen (resolution of $\pm 0.05 \mathrm{~mm}$ ), where the centre thickness is calculated from the scale position of the top of the lens and the position of the top of the probe once the lens has been removed from resting on the probe. To complete all measurements after lens stabilisation typically takes 45 to 50 seconds for a trained operator.
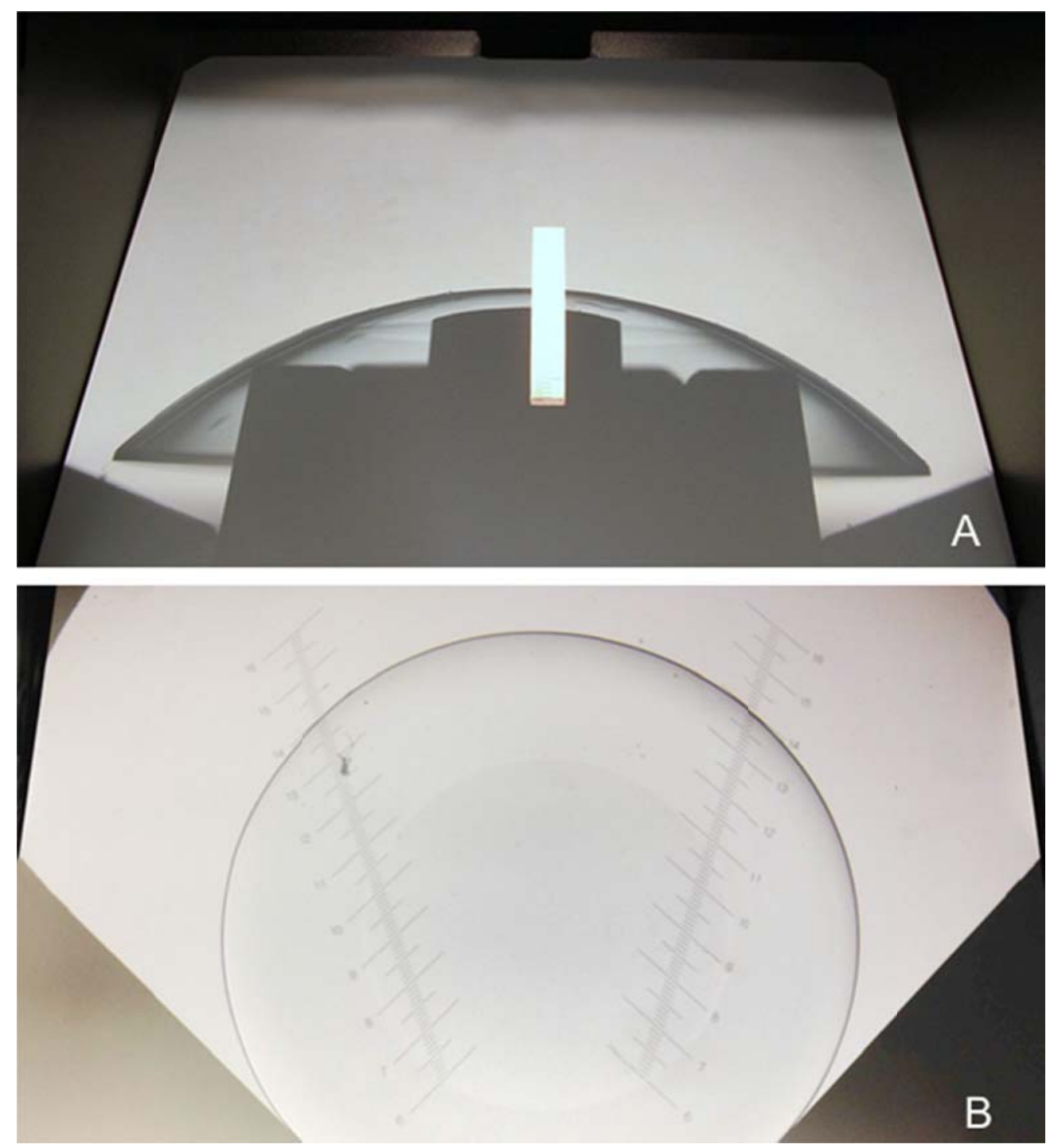

Fig. 1. Illustration of the Optimec JCF measurement methods. A, Illustration of the BOZIR and Centre thickness measurement utilising a cylinder and pillar for measurement of BOZR via the sagittal height, in addition to a visual scale for centre thickness inspection. B, illustration of the diameter measurement cell utilising a graticule with a left and right scale for visual inspection. 


\section{Instrumentation - is830}

The Optimec is830 is a spectral domain OCT-based instrument utilising custom de-warping and image processing software and a unique lens handling solution to ensure suitable hydration and temperature control of lenses. The OCT technology allows for the complete geometric characterisation of the contact lens anterior and posterior surfaces, for samples immersed in a fluid or in air. The test instrument in the current study is a pre-production prototype configured for measurement in saline, but can be adapted to measure samples in air.

A schematic of the instrument is shown in Figure 2. A super luminescent diode (SLD) light source is split between a sample and reference arm by a fibre couple. A Micro-Electrical-Mechanical (MEMs) mirror system is used to scan the light source across a sample, the return reflections are then combined with the return signals from the reference arm and analysed by a spectrometer to characterise the lens. The instrument has a measurement volume of $20 \times 20 \times 6 \mathrm{~mm}$ with an axial resolution in air of $12 \mu \mathrm{m}$ and a lateral resolution of $30 \mu \mathrm{m}$, which can be improved with additional scanning. A camera is used with a beam splitter to image the sample from above.

The contact lens is inserted into the lens cell, and supported on a lens holder. The lens holder contains a number of support arms which the lens rests on and a retaining wall which prevents the lens from moving significantly away from centre. Once the lens is inserted, the operator can position the lens centrally by referencing a camera image from a camera positioned directly above the lens cell. The contact lens rests concave face down on the support structure. The cell is open, allowing for simple insertion and removal of the lens. The lens is immersed in saline (temperature controlled using an Optimec TC20i as described for the JCF) to ensure lens dimensional stability. The lens holder is designed to allow interface free images to be captured across multiple scan lines whilst supporting the lens without lens deformation by distribution of the lens weight across multiple support arms.

The system captures a sequence of one dimensional A-Scans formed of 512 data points representing depth positions based return signals from the sample. The A-Scans are combined into a two dimensional B-Scan to produce a cross-sectional image, with 1024 A-Scans used in the current study, giving images of $1024 \times 512$ pixels. For the current study, a single B-Scan image was captured for every measurement, across a 90 degree meridian (Figure 2). Each B-Scan took 1.07s with a typical positioning time after temperature stabilisation of 8 to $10 \mathrm{~s}$.

To enable geometric parameters to be extracted, the captured images were post-processed to allow the extraction of the anterior and posterior lens edges. Initial image processing was conducted to extract the contact lens region as a binary image, before edge points were extracted based on binary edge detection. After extraction, a custom de-warping procedure based on Snell's law [12] was used to correct for the optical distortions of the saline and sample materials based on the hydrated refractive index of the sample material. Figure 3 illustrates the changes that occur between the initial raw image and the final corrected lens edges, which are used to calculate the geometric parameters of the lens sample. For the current study, BOZR values were calculated using the same method of curvature calculation as the JCF to enable a direct comparison (i.e. across a $10 \mathrm{~mm}$ chord following ISO 18369-3:2006). 


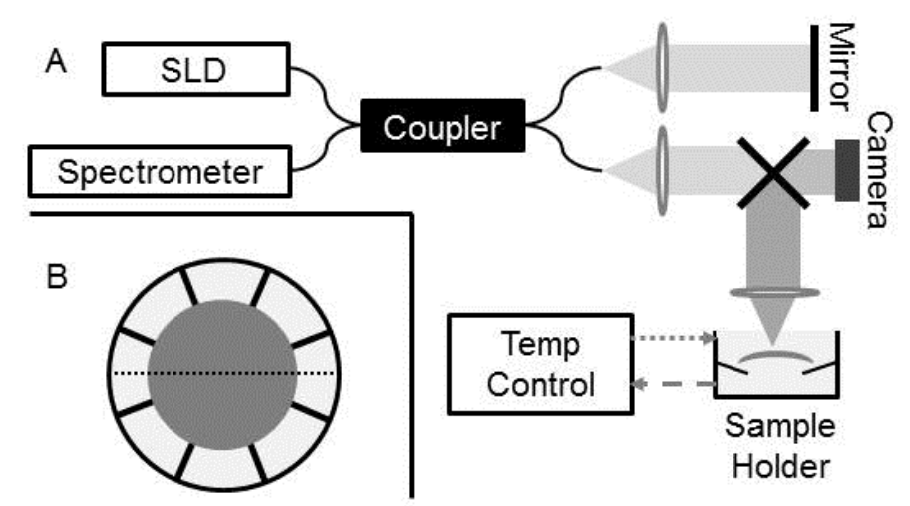

Fig. 2. Experimental setup. A, Optimec is 830 SD-OCT arrangement of SLD light source, reference arm and sample arm, with a camera aligned centrally on the sample holder. Light from the SLD is split by the fibre couple before return signals are combined and received at the spectrometer. Temperature control is used to regulate the saline the lens is immersed in. B, Optimec is830 lens sample holder detail, illustrating the contact lens (blue), lens support arms (solid lines) and example scan lines (dotted).

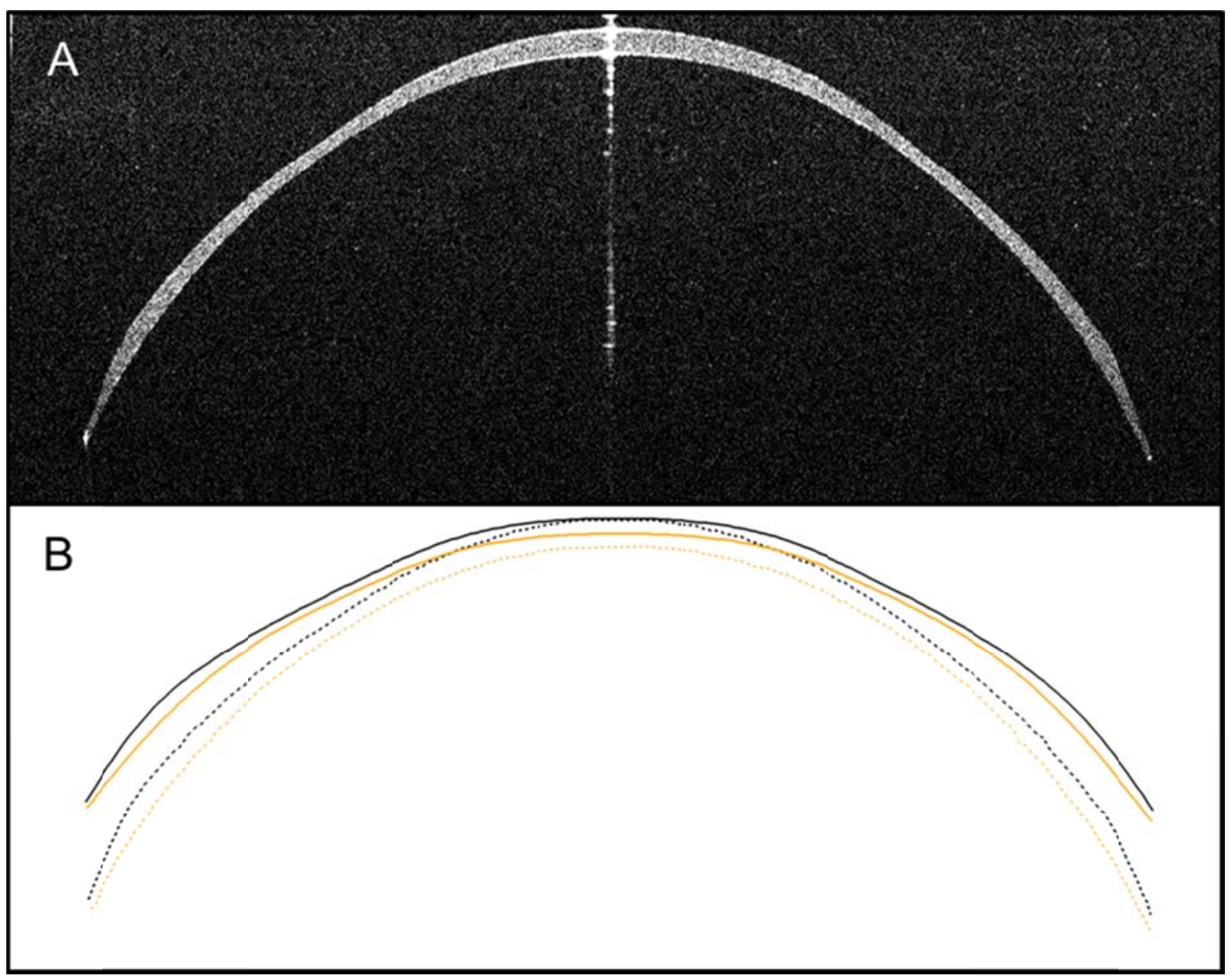

Fig. 3. Illustration of the post processing completing on capture lens scans. $A$, example raw soft contact lens (+6D CooperVision MyDay lens) image captured using the Optimec is830. B, illustration of the extracted edges (dotted) and final de-warped edges (solid) of the lens shown in A. 


\section{Calibration}

The accuracy of an instrument is defined by the difference between measured and actual values. To evaluate this, a calibration piece of known dimensions measured by a certified laboratory can be used. For the is830, a custom calibration piece was commissioned in PMMA and then measured by the National Physical Laboratory (UK), using a co-ordinate measurement machine (CMM) giving xyz coordinates of the anterior and posterior surfaces.

The is 830 was calibrated based on extraction of the edges over a central $8 \mathrm{~mm}$ section where the image was most stable, which could be compared to the CMM xyz coordinates and the control data adjusted until difference between the extracted edges was minimised. For the Optimec JCF, calibration of the curvature measurement was conducted with a glass test piece of known BOZR to calibrate the scale. The glass test piece was placed in the cell and the probe manually adjusted until it touched the spherical surface of the piece. The scale could then be adjusted until it matched the BOZR of the glass piece.

After calibration, the PMMA sample was measured on each instrument using the following procedure. First, the sample was inserted into the measurement fluid (standard saline, controlled to $20^{\circ} \mathrm{C}$ ) and allowed to equilibrate for 10 minutes. Second, a single measurement was completed, following the instrument methods described above. Third, the sample was then removed and placed into its container. Steps one and two were then repeated to obtain a second, independent measurement.

\section{Soft Lens Study}

To conduct a suitable comparison between the JCF and is830, a range of soft contact lenses were used. Two sets of specially commissioned spherical lenses (manufactured by Contamac, UK) were used (all of nominal diameter 14mm), both having 6 lenses ranging in BOZR from $7 \mathrm{~mm}$ to $9.5 \mathrm{~mm}$ in $0.5 \mathrm{~mm}$ steps, with a thin (nominal thickness $0.09 \mathrm{~mm}$ ) and thick (nominal thickness $0.12 \mathrm{~mm}$ ) version of each BOZR step giving 24 lenses in total. The first set was manufactured from a conventional hydrogel (GM Advanced 49\%, Filcon I 1) and the second was manufactured from a silicone hydrogel (Definitve 65, Filcon V 4). A further three commercial lenses were also measured; CooperVision MyDay (Stenfilcon A) in -10D, -3D and +6D powers (nominal diameter $14.2 \mathrm{~mm}$ and BOZR $8.4 \mathrm{~mm}$ ), giving a total of 27 lenses measured on both instruments. For each lens the following procedure was observed. First, the lenses were inserted into glass vials filled with the same saline solution as used in the instruments for at least 24 hours prior to measurement. Second, the lenses were placed in the measurement cell and given 10 minutes stabilisation time to equilibrate at the measurement temperature of $20^{\circ} \mathrm{C}$. Third, the lens was measured following the instrument specific measurement procedure as detailed previously. Fourth, the lens was removed from the instrument and returned to the glass vial. Steps two and three were repeated to give two independent measurements of each lens.

\section{Statistics}

For validity, Bland-Altman [13] plots were created comparing the average measurement of each instrument to the difference between the instruments. The mean difference $(\bar{m})$ was plotted along with the $95 \%$ Confidence internals $(\bar{m} \pm 1.96 s d$, where sd is the standard deviation of the measured 
differences). For repeatability, Bland-Altman plots were created for each measurement parameter comparing the average of the two repeated measures to the difference between the measures, with the mean difference and $95 \%$ confidence intervals calculated.

A repeatability coefficient (CR) was calculated for each parameter using

$$
C R=1.96 \times \sqrt{\frac{\sum\left(r_{1}-r_{2}\right)^{2}}{n-1}}
$$

Where $r_{1}$ and $r_{2}$ are the repeated measurements and $n$ is the number of samples. 


\section{Results}

\section{Calibration}

Figure 4 illustrates the measured (solid lines) and known edge profiles (dashed) of the NPL sample after the adjustments were made to the configuration files. The average deviation between the measured and known edge profiles over the central $8 \mathrm{~mm}$ section was $3.9 \mu \mathrm{m}$ for the anterior edge and $0.9 \mu \mathrm{m}$ for the posterior edge. The deviation from the known edge profile increased at the periphery $(>3.5 \mathrm{~mm}$ from centre), with a maximum deviation of $10.7 \mu \mathrm{m}$ for the anterior edge and $9.9 \mu \mathrm{m}$ for the posterior edge. Table 1 gives the measurement results for the is 830 and JCF measurements of a known test piece after the calibration process was completed.

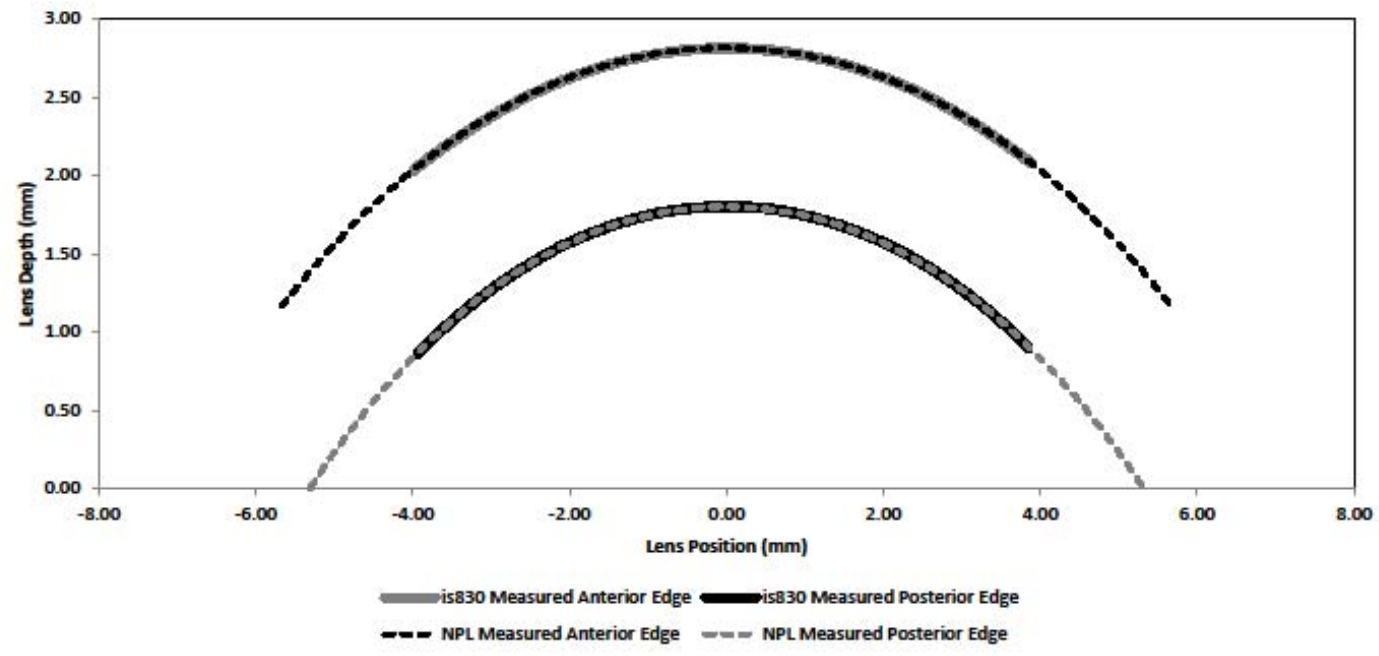

Fig. 4. Illustration of the extracted lens edges of the NPL calibration piece measured on the Optimec is 830 and the edges as measured by the NPL across a central $8 \mathrm{~mm}$ section.

\section{Table 1}

Results of the calibration study comparing the measured data of the calibration piece to the measurements captured by the NPL. The data is averaged across the two measurements, with the standard deviation given for each measurement parameter.

\begin{tabular}{lllllll}
\hline Instrument & \multicolumn{2}{l}{ Centre Thickness $(\mathbf{m m})$} & \multicolumn{2}{l}{ BOZR over } & 10mm Diameter & \multicolumn{2}{l}{ FOZR over 10mm diameter } \\
\hline CMM & 1.013 & \pm 0.0007 & 8.753 & \pm 0.0055 & 10.587 & \pm 0.0055 \\
JCF & 1.0375 & $\pm 0.0125^{\mathrm{c}}$ & 8.725 & \pm 0.025 & - & - \\
Is830 & 1.012 & \pm 0.0005 & 8.712 & \pm 0.007 & 10.577 & \pm 0.006 \\
\hline
\end{tabular}


The results show that both the is 830 and JCF underestimate the BOZR. For the JCF the discrepancy could be attributed to differences in reading when the probe actually touches the underside (hence a larger Standard Deviation), whilst for the is 830 the discrepancies in BOR and FOZR could be attributed to optical distortions at the periphery of the lens, as the standard deviation was very low.

\section{Outcome of the soft lens study}

Figure 5 shows the Bland-Altman comparison between the is830 and JCF for BOZR, Centre thickness and Diameter across the 27 contact lens samples, using the average of the two repeated measurements. The mean difference between the instruments for BOZR was -0.077 with $96 \%$ confidence limits of 0.0740 and -0.228 . For $C T$, the mean difference was -0.010 with confidence limits of 0.001 and -0.0299 . For Diameter, the mean difference was 0.171 , with confidence limits of 0.507 and -0.164 .
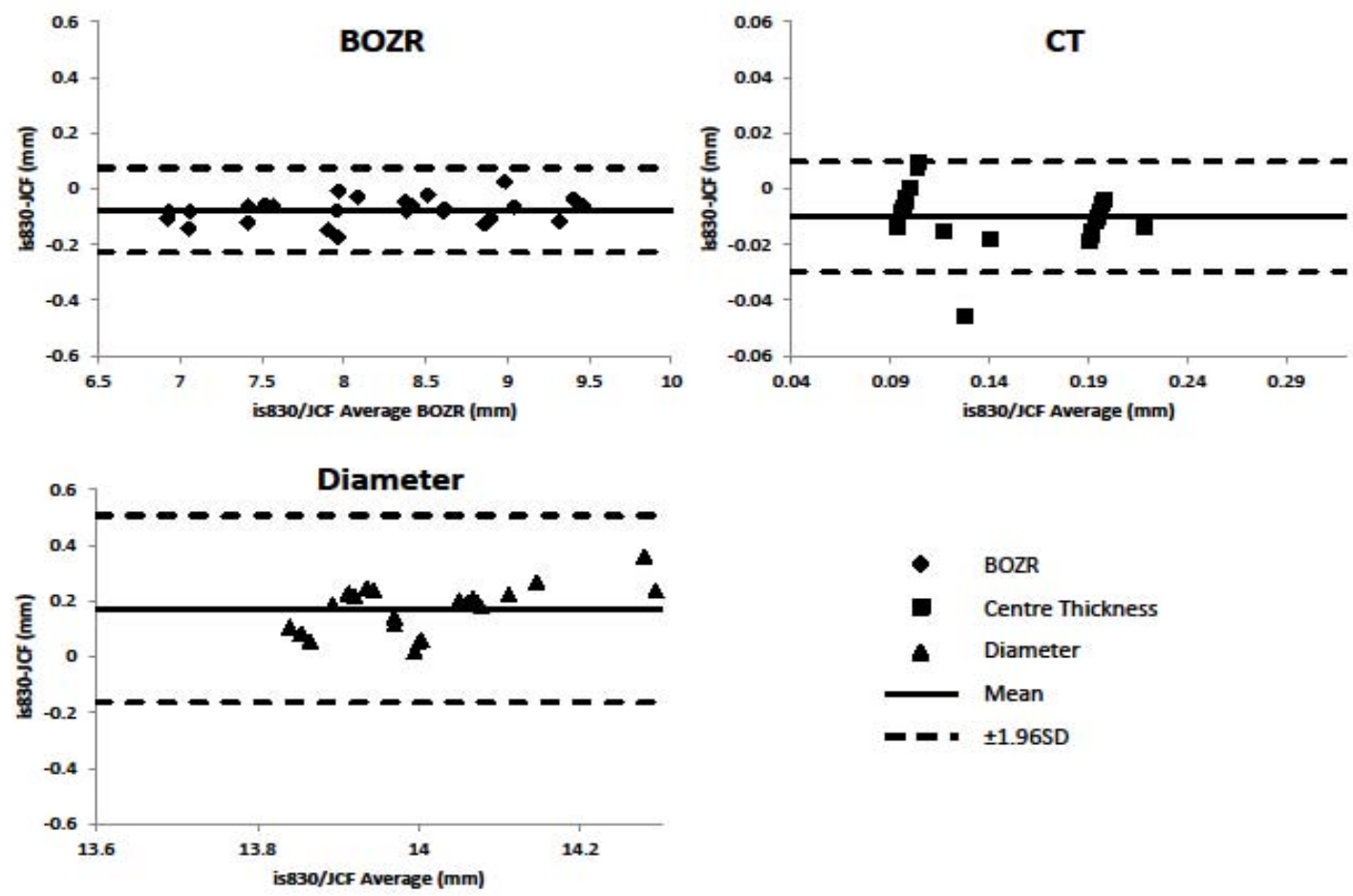

Fig. 5. Bland-Altman comparison of BCOR, CT and Diameter for the JCF and is 830 , with mean $95 \%$ confidence interval lines

To analyse the differences between the instruments further, Fig. 6 shows a direct comparison between the two instruments in terms of repeatability of the independent measurements for BOZR, CT and Diameter, with the repeatability coefficients given in Table 2.

The results show that the is 830 is significantly more repeatable than the JCF for centre thickness and BOZR, but has a similar value for diameter. In addition, there is significantly less bias between measurements for BOZR (0.0012 compared to 0.0296) and CT (0.0003 compared to 0.0037), although the JCF had less bias than the is 839 for diameter $(0.0009$ compared to 0.0036$)$. For the 
remaining parameters that are able to be measured on the is 830 , the repeatability coefficients compare well with the main parameters (Table 2).
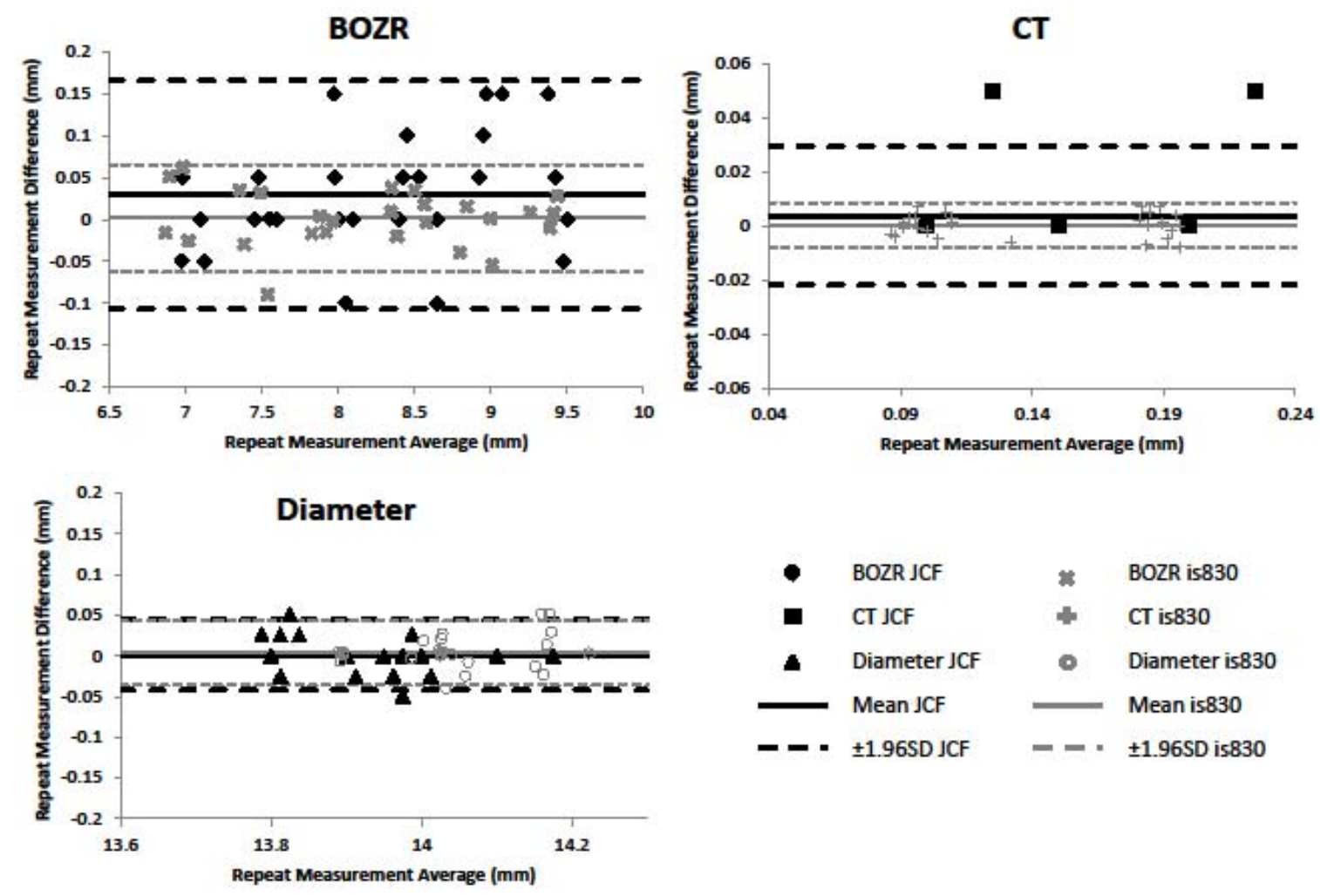

Fig. 6. Bland-Altman repeatability comparison for BOZR, CT and Diameter for the JCF and is 830 with mean difference and $95 \%$ confidence interval lines.

Table 2: The repeatability coefficients (CR) for the measurement parameters on both the JCF and is830, calculated across the two repeated measures of the 27 contact lens samples

\begin{tabular}{ccc}
\hline Parameter & CR, JCF & CR, is830 \\
\hline BOZR & 0.151 & 0.065 \\
CT & 0.033 & 0.008 \\
Diameter & 0.044 & 0.041 \\
Posterior & - & 0.072 \\
Sagittal Depth & - & 0.091 \\
FOZR & - & \\
\hline
\end{tabular}

Figure 7 illustrates the raw image data able to be captured on the is 830 , where it can be seen that an edge to edge cross-section of the lenses can be extracted. Hence, the is830 is able to extract a range of additional lens measurements such as the thickness and sagittal depth at multiple positions away from centre (Figure 8 ) in addition to the FOZR (Table 2). 

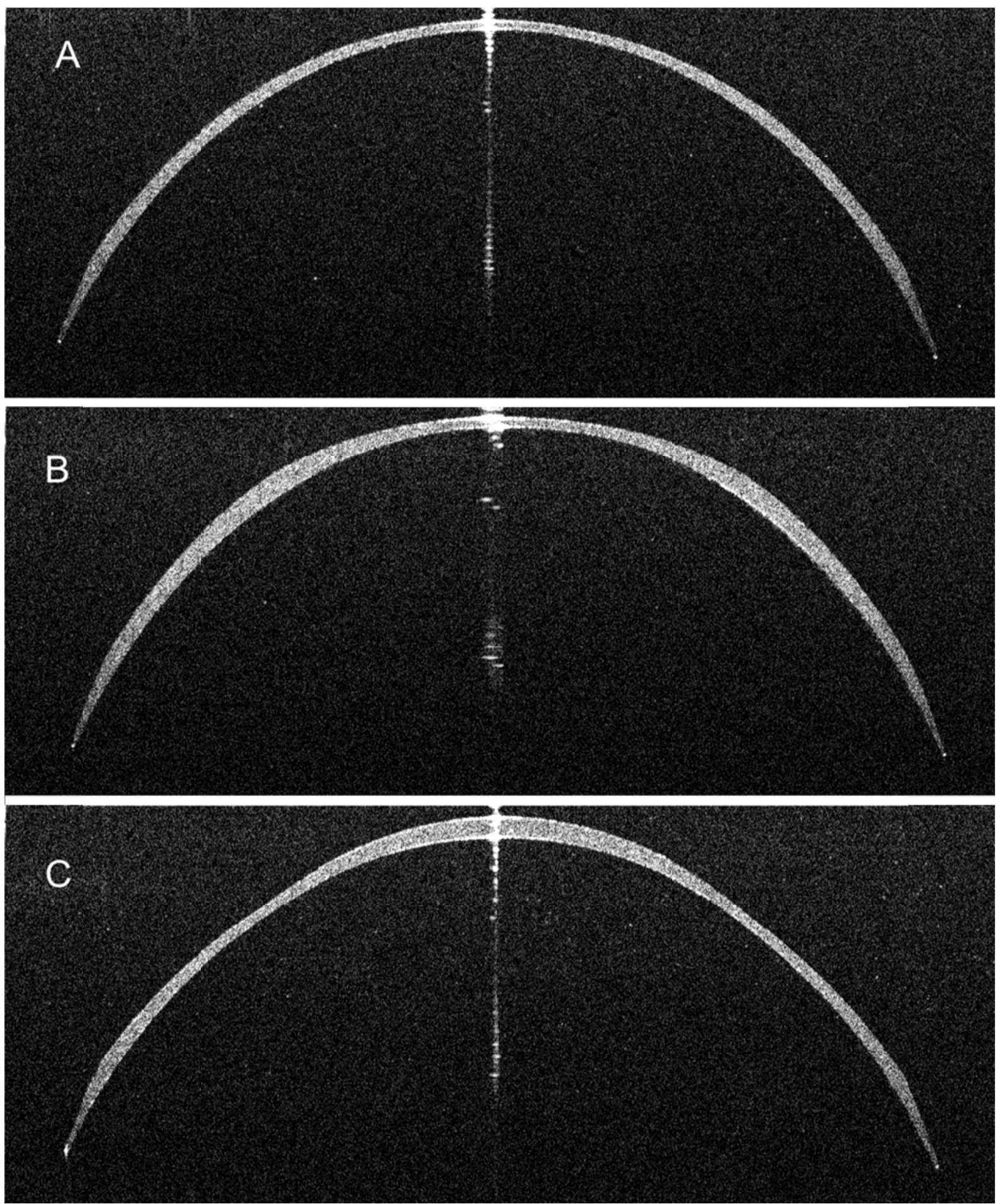

Fig. 7. Comparison between examples of the raw, uncorrected image data of the three CooperVision MyDay lenses immersed in saline as measured in the study using the is 830 . A $-3 D$ lens, B $-10 D$ lens, C $+6 D$ Lens 

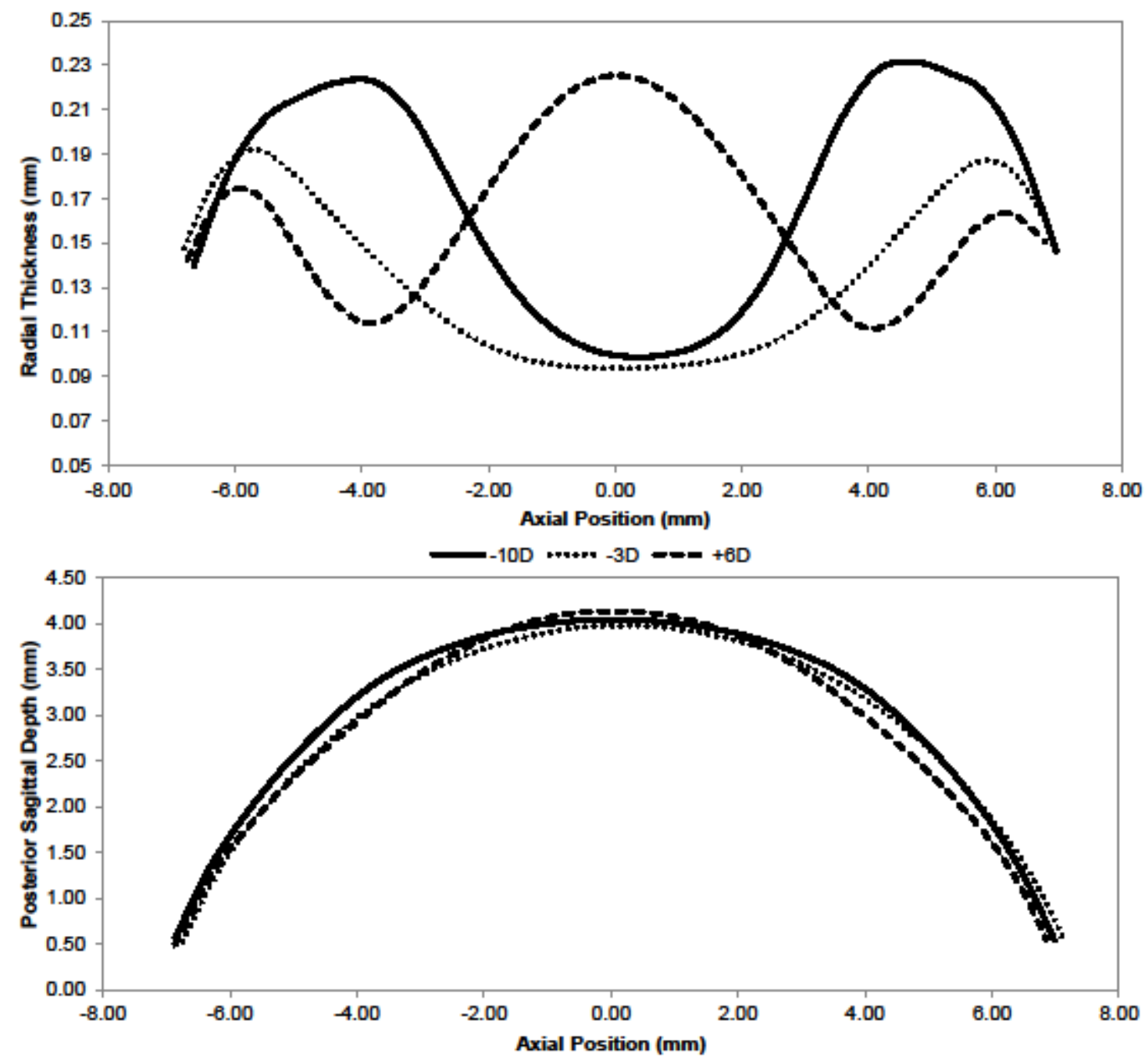

Fig. 8. Comparison of the extracted thickness profiles (Top) and posterior sagittal height profiles (Bottom) for the three CooperVision MyDay lenses as measured using the is830. Each data set is the average of two measurements. 


\section{Discussion}

Optical Coherence Tomography is becoming an increasingly valuable tool for industrial inspection across a wide number of applications and initial studies with contact lenses have shown that it is relevant to contact lens inspection [8-11]. The ability to characterise the entire anterior and posterior contact lens surface, and therefore extract geometric measurement data, would be very useful for quantifying complex lens shapes, as this is something very difficult to achieve with existing contact lens inspection equipment and missing from the current ISO standards. The increasing complexity of soft and rigid contact lens would benefit from an inspection system that is easily adopted into current measurement laboratories that can measure multiple parameters in a temperature controlled and hydrated manner in a single instrument.

This study compares a widely used geometric inspection instrument, the Optimec JCF, to an OCT based geometric inspection instrument, the Optimec is830, currently under development. The results presented in the current study have illustrated how the measurement capability of the is 830 compares well with the JCF for Diameter and offers significant improvement for BOZR and CT. The is 830 also presents the opportunity to investigate multiple geometric parameters of contact lenses in a single instrument, removing potential measurement discrepancies that can be introduced in measuring single lenses across multiple instruments. In addition, the OCT methodology allows the measurement of geometric data, such as sagittal depth and thickness at the centre and periphery of the lens.

There are some discrepancies between the instruments, in particular for BOZR. As the JCF relies on subjective operator judgement to determine the moment the probe touches the underside of the lens, this may account for the larger standard deviation and discrepancy in the BOZR and CT values for the PMMA and soft lens samples. In addition, due to the difference in support methods, there is the potential for a difference in how a soft lens may sag during the measurement process, potentially affecting repeatability on soft lens samples (Figure 6). The good repeatability for the is 830 would suggest the discrepancies in BOZR and FOZR measurements on the PMMA sample could be attributed to the apparent increase in deviation away from the centre of the lens (Figure 4), which could also impact on the measurement difference between the instruments for the soft lenses (Figure 5). For diameter, it is unlikely that the differences can be attributed to the repeatability as both instruments have very similar results (Figure 6). However, Figure 5 indicates a bigger differential between the instruments as the diameter increases, which could be attributed to the increase in deviation at the periphery of the lens as shown on the PMMA sample for the is 830.

Although the is 830 has demonstrated the potential for OCT to be used for contact lens inspection by matching the performance of a widely used instrument, the current study has also highlighted the need to improve on the measurement accuracy of the is 830 over a larger area (i.e. for curvature and diameter). In addition, although the usage of an open wet cell simplifies the insertion and removal of the lens, centration of the lens is subjectively optimised potentially contributing to the errors in measurement of curvature. However, the benefits in terms of measurement time (typically between 10 - 15 seconds for the is 830 compared to $45-50$ seconds for the JCF) would strongly suggest that it is beneficial to retain a methodology similar to this, rather than moving to complex methods, so ensuring suitability for a production environment. 
The current study has demonstrated that the measurement capability of an OCT based instrument which is currently able to match a widely used method and offers a number potential improvements over existing methods. To establish whether OCT could be appropriate to be included in future ISO standards, further validation of a commercially available instrument is required to assess repeatability and reproducibility on both spherical and non-spherical lenses.

\section{Conflict of interest}

Ben Coldrick is an associate in the Knowledge Transfer Partnership between Aston University and Optimec Limited.

\section{Acknowledgments}

The project was funded by Innovate UK as part of a Knowledge Transfer Partnership (KTP009287) between Aston University (Birmingham, UK) and Optimec Limited (Malvern, UK). 


\section{References}

[1] BSI. BS EN ISO 18396-3:2006. Ophthalmic optics - Contact lenses - Part 3: Measurement methods: BSI; 2006.

[2] Marcus Ma, Hadcock KJ, Gibson DS, Herbrand ME, Ignatovich FV. Precision interferometric measurements of refractive index of polymers in air and liquid. Optifab 2013: SPIE; 2013. p. 88841L1-L10.

[3] Plainis S, Atchison DA, Charman WN. Power Profiles of Multifocal Contact Lenses and their Interpretation. Optometry and Vision Science. 2013;90:1066-77.

[4] Young G. Daily disposable soft lens diameters. Optician. 2008;235:38-40.

[5] van der Worp E, Mertz C. Sagittal height differences of frequent replacement silicone hydrogel contact lenses. Contact Lens and Anterior Eye. 2015;38:157-62.

[6] Lira M, Pereira C, Real Oliveira MECD, Castanheira EMS. Importance of contact lens power and thickness in oxygen transmissibility. Contact Lens and Anterior Eye. 2014;38:120-6.

[7] Swarbrick Ha. Orthokeratology review and update. Clinical and Experimental Optometry. 2006;89:124-43.

[8] Shen M, Wang MR, Wang J, Yuan Y. Entire contact lens imaged in vivo and in vitro with spectral domain optical coherence tomography. Eye \& Contact Lens. 2010;36:73-6.

[9] Zhu D, Shen M, Li Y. Parameters measurement of rigid gas permeable contact lens based on optical coherence tomography. 6th International Symposium on Advanced Optical Manufacturing and Testing Technologies: Optical Test and Measurement Technology and Equipment: SPIE; 2012. p. 84171D1-D6.

[10] Wolffsohn JS, Drew T, Dhallu S, Sheppard A, Hofmann GJ, Prince M. Impact of soft contact lens edge design and midperipheral lens shape on the epithelium and its indentation with lens mobility. Investigative Ophthalmology \& Visual Science. 2013;54:6190-7.

[11] Karnowski K, Grulkowski I, Mohan N, Cox I, Wojtkowski M. Quantitative optical inspection of contact lenses immersed in wet cell using swept source OCT. Optics Letters. 2014;39:4727-30.

[12] Podoleanu A, Charalambous I, Plesea L, Dogariu A, Rosen R. Correction of distortions in optical coherence tomography imaging of the eye. Physics in Medicine and Biology. 2004;49:1277-94.

[13] Bland JM, Altman DG. Measuring agreement in method comparison studies. Statistical Methods in Medical Research. 1999;8:135-60. 